\title{
A Method to Develop a Personalized Pattern of Pant from 3D Scanning Data
}

\author{
Junqiang SU*1, Ying KE ${ }^{1}$, Bingfei $\mathrm{GU}^{2}$, Caiyuan KUANG ${ }^{3}$ \\ ${ }^{1}$ School of Textile and Clothing, Jiangnan University, Jiangsu, China; \\ ${ }^{2}$ Fashion College, Zhejiang Sci-Tech University, Zhejiang, China; \\ ${ }^{3}$ College of Art, Jinling Institute of Technology, Jiangsu, China
}

DOI: $10.15221 / 17.121 \quad$ http://dx.doi.org/10.15221/17.121

\begin{abstract}
A method was demonstrated to develop a personalized pattern of pant from 3D scanning data. There were three main steps to complete the purpose: 1) realization of individuality of a body; 2) description of distribution of ease allowance at characteristic features; and 3) a method to alter a basic pant pattern to develop a personalized one.
\end{abstract}

Keywords: apparel customization, 3d body scanning, body feature, pattern making

\section{Introduction}

With the development of technologies, consumers appear to purchase a clothing fits well made by customization with higher quality and lower price. With the development of Internet and E-commerce, costs for materials and transaction to manufacture have reduced greatly. However, the demand for customization is rising significantly. One promising trend in the filed of appreal manufacturing is increasingly growing up which requires manufacturers to find a way to meet the rising requirement and change themselves from mass-production to MTM (Made to Measure), or more advanced, masscustomization.

In the past decade, new technologies, such as 3D body scanning for anthropometry, big data for analysis of body types, intellgent CAD for pattern-making, flexible manufacturing systems, and virtual try-on technology for fitting, have greatly pushed forward the mass-customization operations ${ }^{[1-5]}$. Among them, the technology to make a personalized pattern still remains the core of customization.

An personalized pattern-making system is a vital part of mass customization because it solves the problem of how to produce a well-fitted apparel pattern according to a customers' figure ${ }^{[4]}$. One main purpose of using the customized system is to provide a prompt way to generate individualized apparel patterns directly from digital body data. As summarized by Yang, et. al. ${ }^{[7]}$, there are two main ways to implementing the purpose.

The first way is to modify a ready-to-wear pattern using a certain grading rule. Modification of garment pattern is an essential step in producing a good-fit clothing, which can be further divided into two methods. The first uses mathematical or experience-based formula to change shape of curves in a basic pattern to fit a specific body, which is usually used in traditional CAD system. The key is to modify a pattern in conjunction with sizing and alteration tables that are designated by a national or industrial standard. The second method is based on Artificial Intellgent (Al) technologies, artificial neural networks (ANN) and fuzzy logic (FL) are the most relevant. ANN has been proven to be feasible and useful in developing models for nonlinear systems. By the view of ANN, an clothing pattern is a set of elements including points, lines, curves and sizing. Pattern making is just to decide the setting according to individual body measurements and fabric properties. Similiarly, FL provides a simple way to arrive at a definite conclusion based upon vague, ambiguous, imprecise, noisy, or missing input information. By the view of FL, many problems, such as the fit or the ease of the pattern, cannot be described precisely but is suitable to deal with FL technology. Summarily, the Al techniques depends on parameters that describe the topological relationships between points, lines and curves in pattern making.Further, the value of the parameters usually determined by an operator's experience instead of ideas of taking advantage of 3D data of a body.

Another way is to generate a personalized pattern through flattening a 3D garment by a computer ${ }^{[8-13]}$. It is logical to think of that a well-fitted pattern can be obtained by flattening a 3D model which was built up from a person's scanning data. In the development of apparel CAD systems, considerable attention has been paid to the flattening techniques. Given a 3D free form surface and the material properties, the counterpart flat patterns can be generated through the flattening process. The main advantage of this method is to generates pattern pieces directly from a 3D virtual model, which retains the shape of an individual figure in developing a pattern. However, it is hard to integrate this virtual pattern into the pattern-making systems that have already been in operation for decades, and the practicability and accuracy of the pattern by this method needs to be improved. 
According to the relationship between features of a body and curves in a pattern of clothing, a female's body surface can be seen as a tightest clothing which covers the body without any ease, and a wellfitted clothing can be looking as an overlap with a proper ease upon a basic pattern. Thus, there are three main projects need to be revealed: 1) how to develop a personalized basic pattern from scanning data; 2) how to add an ease allowance to features' location in a pattern; and 3) how to develop a personalized pattern using the result in the former two projects.

\section{Development of a personalized basic pattern}

According to principle of "body-pattern" in Fig. 1 and reference [14], we took a body surface as a tighest clothing and locations of features as frame of a pattern. Moveover, according to the inherent relation between a body and a clothing, the shape of a body's feature determines the shape of a curve in the pattern, individual features lead to individualized curves and subsequently individulized pattern. Therefore, analysis of a body's features become an essential work to realize individuality in a clothing pattern.

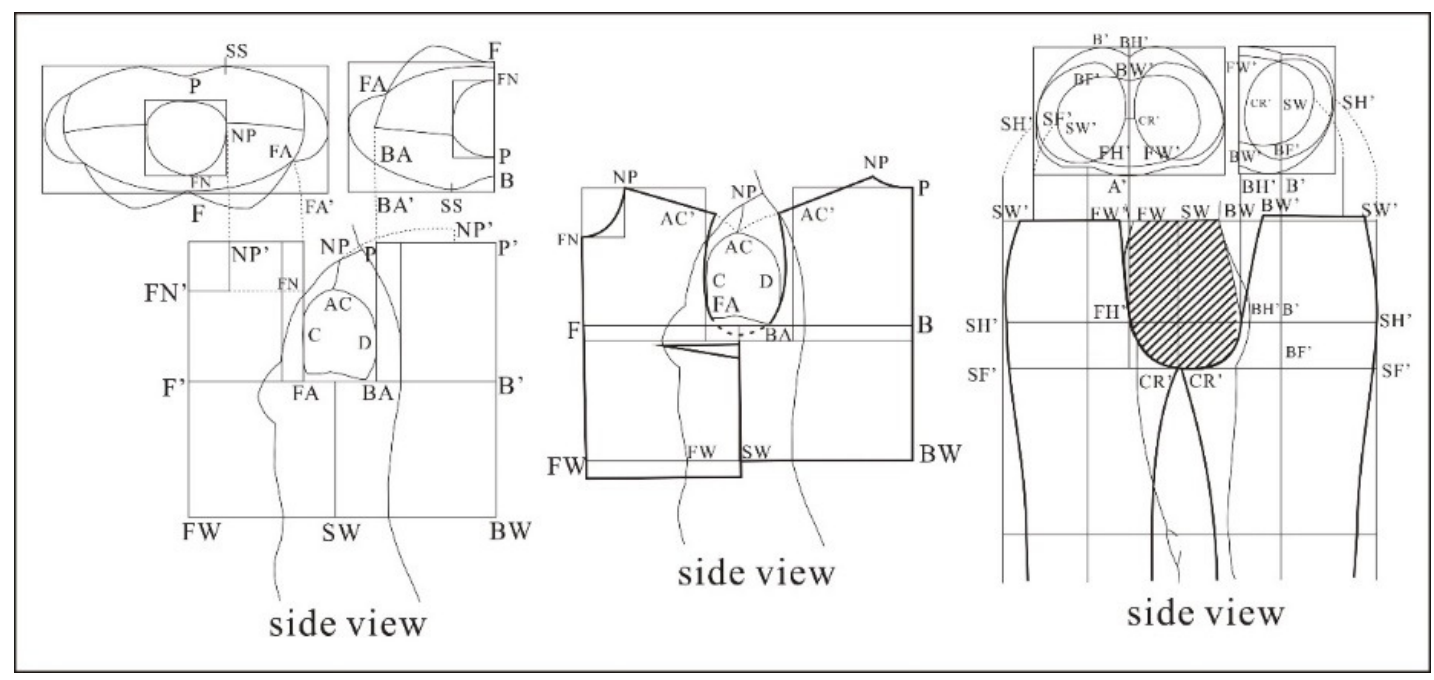

Fig. 1. Relationship between body and apparel pattern.

In this paper, only the lower body and the pant pattern was selected to be studied. The individualization was divided into three parts- the individilities on height, on girth and on crotch, rules were studied for each part respectively. Correspondingly, a body's 3D feature was also divided into height, width, and thickness to measure. The girths such as waist, abdomen, hip, thigh, knee and ankle, were carried out according to ASTM 5219-94d. The Width (Thickness) of a certain feature is the width (thickness) in front (side) view at the feature's position, and projected length if the girth is inclined. The length of Central Front/Back Line and Front/Back Crotch was measured along the surface of the body. Moreover, in side view, we defined lateral line as a line which across midpoint of thickness of waist and midpoint of thickness of calf. Based on the lateral line, features were divided into Front and Back parts. Please see more details in Figure 2.
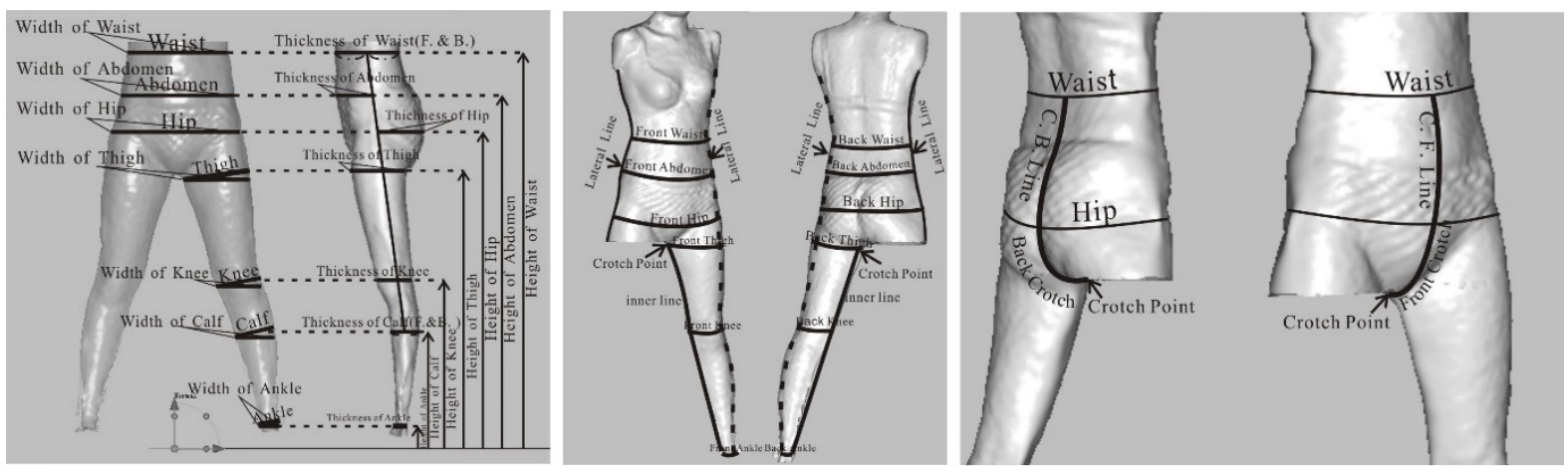

Fig. 2. Method to measure a body surface. 


\subsection{Method to realize individuality of a body}

As metioned before, how to describe the individuality of a body is the key to develop a personalized pattern. Our method takes two main steps. The first is to describe and formulate the features of a body with parameters or formulas relating to the shape of some elements, such as points, lines, curves or angles, in a pattern. The second is to classify the parameters or formula into grades that provide different ranges to fit the variety of bodies, and to provide values for adjusting the elements to be individual. Here, we took only an example of girth to illustrate the methodology.

A shape of cross-section at a characteristic position gives the most exact information for body figure and determines length and shape of curves in structure of a pant pattern. So the ratio of thickness and width was adopted to reflect a feature's individuality as $R G_{\text {feature }}=\frac{\text { thickness of feature }}{\text { width of feature }}$, in which the subscript indicates the feature's name relevant.

The thickness and the width of cross section at each feature were measured and the ratio was calculated respectively. Subsequently, the maximum and minimum of ratio were found and arranged with a 0.05 differece. Further, the quantity which belongs to a certain grade was counted and the result showed in Table 1.

Table 1. Distribution of ratio for girths

\begin{tabular}{|c|c|c|c|c|c|c|}
\hline Grade & 1 & 2 & 3 & 4 & 5 & 6 \\
\hline Grade Value & $0.51-0.55$ & $0.56-0.60$ & $0.61-0.65$ & $0.66-0.70$ & $0.71-0.75$ & $0.76-0.81$ \\
\hline Waist & 8 & 60 & 112 & 146 & 78 & 12 \\
\hline Abdomen & 6 & 71 & 120 & 132 & 75 & 11 \\
\hline Hip & 12 & 105 & 167 & 102 & 27 & 3 \\
\hline Thigh & 23 & 126 & 132 & 85 & 31 & 19 \\
\hline Knee & 45 & 188 & 100 & 62 & 16 & 5 \\
\hline Calf & 12 & 86 & 152 & 115 & 42 & 9 \\
\hline
\end{tabular}

It can be seen from Table 1 that the ratio mainly concentrates on one or two grade(s) for each feature. It is logically to explore the exact relationship among the width, the thickness and the girth. Therefore, correlation analysis among width and thickness of feature and girth of a cross section was made. Table 2 shows the result that all of the correlation coefficient $R^{2}$ are relatively high, and sig.(2-tailed) are 0.000 $<0.01$, which illustrates that the variables have significant correlationship. Thus, it is feasible to get the relationship among width and thickness of a featurres and its girth by method of regression.

Table 2. Correlation analysis among width and thickness and girth of features

\begin{tabular}{|c|c|c|c|c|}
\hline & \multicolumn{2}{|c|}{ Width of waist } & \multicolumn{2}{c|}{ Thickness of front waist } \\
\hline & $\mathrm{R}^{2}$ & Sig.(2-tailed) & $\mathrm{R}^{2}$ & Sig.(2-tailed) \\
\hline Front waist & $0.898^{*}$ & 0.000 & $0.865^{*}$ & 0.000 \\
\hline Front abdomen & $0.886^{*}$ & 0.000 & $0.849^{*}$ & 0.000 \\
\hline Front hip & $0.822^{*}$ & 0.000 & $0.806^{*}$ & 0.000 \\
\hline & \multicolumn{2}{|c|}{ Width of waist } & \multicolumn{1}{c|}{ Thickness of back waist } \\
\hline & $\mathrm{R}^{2}$ & Sig(2-tailed) & $\mathrm{R}^{2}$ & Sig.(2-tailed) \\
\hline Back waist & $0.856^{*}$ & 0.000 & $0.917^{*}$ & 0.000 \\
\hline Back abdomen & $0.801^{*}$ & 0.000 & $0.883^{*}$ & 0.000 \\
\hline Back hip & $0.787^{*}$ & 0.000 & $0.836^{*}$ & 0.000 \\
\hline & \multicolumn{2}{|c|}{ Width of thigh } & \multicolumn{2}{c|}{ Thickness of thigh } \\
\hline Thigh & $\mathrm{R}^{2}$ & Sig(2-tailed) & $\mathrm{R}^{2}$ & Sig.(2-tailed) \\
\hline Knee & $0.790^{*}$ & 0.000 & $0.793^{*}$ & 0.000 \\
\hline Calf & $0.766^{*}$ & 0.000 & $0.720^{*}$ & 0.000 \\
\hline
\end{tabular}

Scatter diagrams among width and thickness of each feature and its girth were made to observe trend of distribution. There were two examples for explanation, the front waist and thickness of front waist in Fig. 3 (a) and the front waist and width of waist in Fig. 3 (b). 


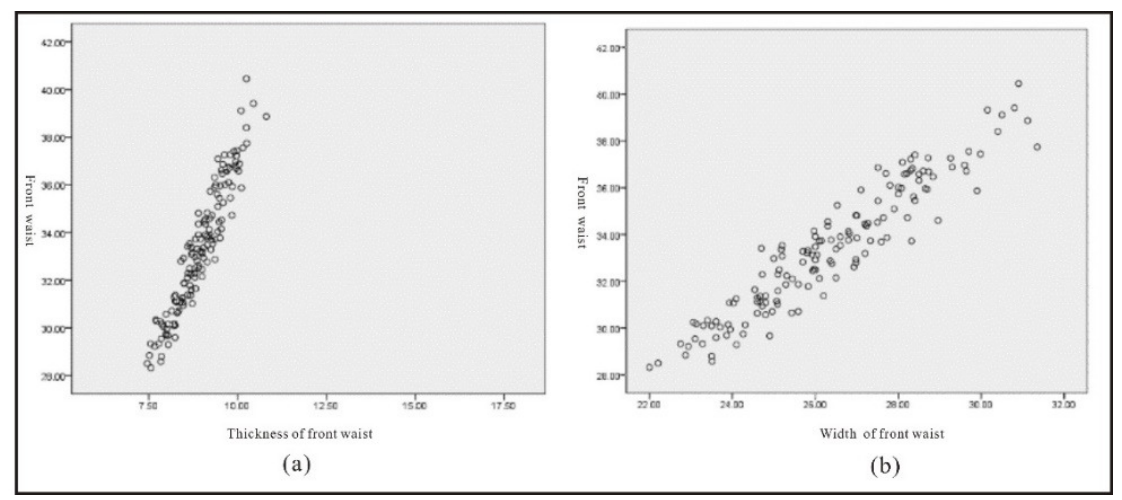

Fig. 3. Scatter diagram among Thickness (a)/Width (b) of front waist and Front Waist(in grade 4)

Based on the scatter diagram and the correlation analysis, linear regressions were bulit up as shown in formula $(1) \sim(2)$.

$$
\begin{aligned}
& F W=\mathrm{a}_{0}+\mathrm{a}_{1} * T_{f w}+a_{2} * W \\
& \mathrm{BW}=b_{0}+b_{1} * T_{b w}+b_{2} * W
\end{aligned}
$$

Note: FW , BW- Front/Back Waist; $\mathrm{a}_{0}, b_{0}$-Constant; $\mathrm{a}_{1}, a_{2}, b_{1}, b_{2}$-Regression coefficient; $T_{f w}$ - Thickness of front waist; $T_{b w}$ - Thickness of back waist; $W$ - Waist.

The constant and the regression coefficient were obtained by regression analysis at each characteristic postion. Because the similar procedure of regression, there was only an example of waist for illustration in Table 3 which shows the result of regression.

Table 3. Constant and the regression coefficient at waist

\begin{tabular}{|c|c|c|c|c|c|c|}
\hline Grade & $a_{0}$ & $a_{1}$ & $a_{2}$ & $b_{0}$ & $b_{1}$ & $b_{2}$ \\
\hline 1 & -32.382 & 1.406 & 0.937 & -96.844 & 2.074 & 1.084 \\
\hline 2 & -3.024 & 1.285 & 0.862 & -42.287 & -0.088 & 1.427 \\
\hline 3 & 8.468 & 1.493 & 0.743 & -52.825 & 1.816 & -0.991 \\
\hline 4 & 9.968 & 0.626 & 1.043 & -51.837 & 0.642 & 1.375 \\
\hline 5 & -8.327 & 1.782 & 0.681 & -42.454 & 1.147 & 1.157 \\
\hline 6 & 1.549 & -0.261 & 1.429 & -51.654 & 2.327 & 0.787 \\
\hline
\end{tabular}

An error check for regression at each feature was carried out. We arranged the absolute error with $1 \mathrm{~cm}$ a grade and counted the number falled into a certain grade, the result showed in Table 4 . It shows a good and high concentration for the front and back features, and most participantors have an absolute error within $1 \mathrm{~cm}$, which is reliable for body measuring and further analysis.

Table 4. Error check for regressions on girths

\begin{tabular}{|c|c|c|c|c|c|}
\hline \multirow{2}{*}{ Front waist } & & {$[0,1] \mathrm{cm}$} & {$[1,2] \mathrm{cm}$} & {$[2,3] \mathrm{cm}$} & $>3 \mathrm{~cm}$ \\
\hline \multirow{2}{*}{$\begin{array}{c}\text { Front } \\
\text { abdomen }\end{array}$} & Quantity & 307 & 91 & 16 & 2 \\
\cline { 2 - 6 } & Proportion & $73.8 \%$ & $21.9 \%$ & $3.8 \%$ & $0.5 \%$ \\
\cline { 2 - 6 } Front hip & Quantity & 375 & 40 & 1 & 0 \\
\cline { 2 - 6 } & Quantity & $90.1 \%$ & $9.6 \%$ & $0.2 \%$ & $0.0 \%$ \\
\hline \multirow{2}{*}{ Back waist } & Proportion & $78.1 \%$ & $19.2 \%$ & $2.2 \%$ & $0.5 \%$ \\
\cline { 2 - 6 } & Quantity & 328 & 80 & 7 & 1 \\
\hline \multirow{2}{*}{ Back abdomen } & Proportion & $78.8 \%$ & $19.2 \%$ & $1.7 \%$ & $0.2 \%$ \\
\cline { 2 - 6 } & Quantity & 369 & 46 & 1 & 0 \\
\hline \multirow{2}{*}{ Back hip } & Qroportion & $88.7 \%$ & $11.1 \%$ & $0.2 \%$ & $0.0 \%$ \\
\cline { 2 - 6 } & Proportion & $83.4 \%$ & $14.7 \%$ & $1.2 \%$ & $0.7 \%$ \\
\hline \multirow{2}{*}{ Thigh } & Quantity & 185 & 141 & 61 & 29 \\
\cline { 2 - 6 } & Proportion & $44.5 \%$ & $33.9 \%$ & $14.7 \%$ & $7.0 \%$ \\
\hline \multirow{2}{*}{ Knee } & Quantity & 228 & 122 & 38 & 28 \\
\cline { 2 - 6 } & Proportion & $54.8 \%$ & $29.3 \%$ & $9.1 \%$ & $6.7 \%$ \\
\hline \multirow{2}{*}{ Calf } & Quantity & 300 & 92 & 23 & 1 \\
\cline { 2 - 6 } & Proportion & $72.1 \%$ & $22.1 \%$ & $5.5 \%$ & $0.2 \%$ \\
\hline
\end{tabular}


When a certain person was scaned and the $R G_{\text {feature }}$ was calculated, a grade could be chosen according the grade value in Table 1. Subsequently, a regression in Table 3 was selected according to the grade value, therefore the regression coefficient was settled down. Finally, because the Waist and Thickness of front/back waist were easy to measure and obtained, the Front/Back Waist were predicted rapidly, which was relative to the length of front/back waist in a pant pattern.

Similar method were carried out at each characteristic positions, so the individulity of a body were described in a personalized way, in other words, there are alternatives for pattern making to choose.

\subsection{Distribution of ease allowance at characteristic features}

Many research efforts have been taken in the area of ease assessment and determination. Zhang et al. ${ }^{[15]}$ studied the distribution of the distance ease in the bust section at different radial angles. They scanned an unclothed dummy and the same dummy clothed with shirts separately and set a radial coordinate system on the shirt-body circumference at bust location for quantitative analysis. Our experiment was similar with Zhang's but different with the coordinate system for measuring.

A female student in our university was chosen as an experimental object. Her body sizes conform to the Chinese standard on dummy mannequin (GB/T 1335.2-2008), which represents the characteristics of body shape of East Asian females. The height, bust, waist and hip lines of the object are 163, 84, 67 and $90 \mathrm{~cm}$, respectively. The style of garment samples was suit pant, designed with front and back darts along the waist band without any pockets or other accessories, as shown in Figure 4 . The size of the pants was divided into three categories: Tight Fit- ease allowance from 0 to $4 \mathrm{~cm}$, Fit- ease allowance from 6 to $10 \mathrm{~cm}$ and Loose- ease allowance from 12 to $16 \mathrm{~cm}$, which include almost all of possible ease allowance for ready-to-wear pants given by designers. We made one garment sample for each of the "Tight Fit" and "Loose" categories and two for the "Fit" category. More information about the sizes of these pants is shown in Table 5.

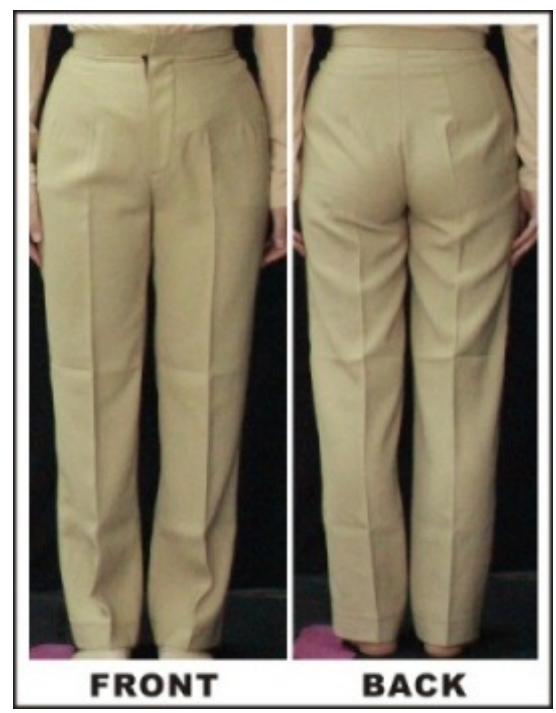

Fig. 4. Pant style for experiment.

Table 5. Sizes of sample pants.

\begin{tabular}{|c|c|c|c|c|}
\hline \multicolumn{2}{|c|}{ Ease Allowance $(\mathrm{cm})$} & Sample & Waist $(\mathrm{cm})$ & Hip $(\mathrm{cm})$ \\
\hline Tight Fit & $0 \sim 4$ & $\# 1$ & 67 & 94 \\
\hline \multirow{2}{*}{ Fit } & \multirow{2}{*}{$6 \sim 10$} & $\# 2$ & 67.5 & 96 \\
\cline { 2 - 5 } & $12 \sim 16$ & $\# 3$ & 68 & 100 \\
\hline Loose & & $\# 4$ & 68.5 & 104 \\
\hline
\end{tabular}

Next, the unclothed and clothed body scanning data were obtained and overlaped. Thus, cross-sections could be made to get the pant-body circumferences at various locations. Figure 5 displays the pantbody circumferences of hip, abdomen, thigh and knee with four different ease allowances. 


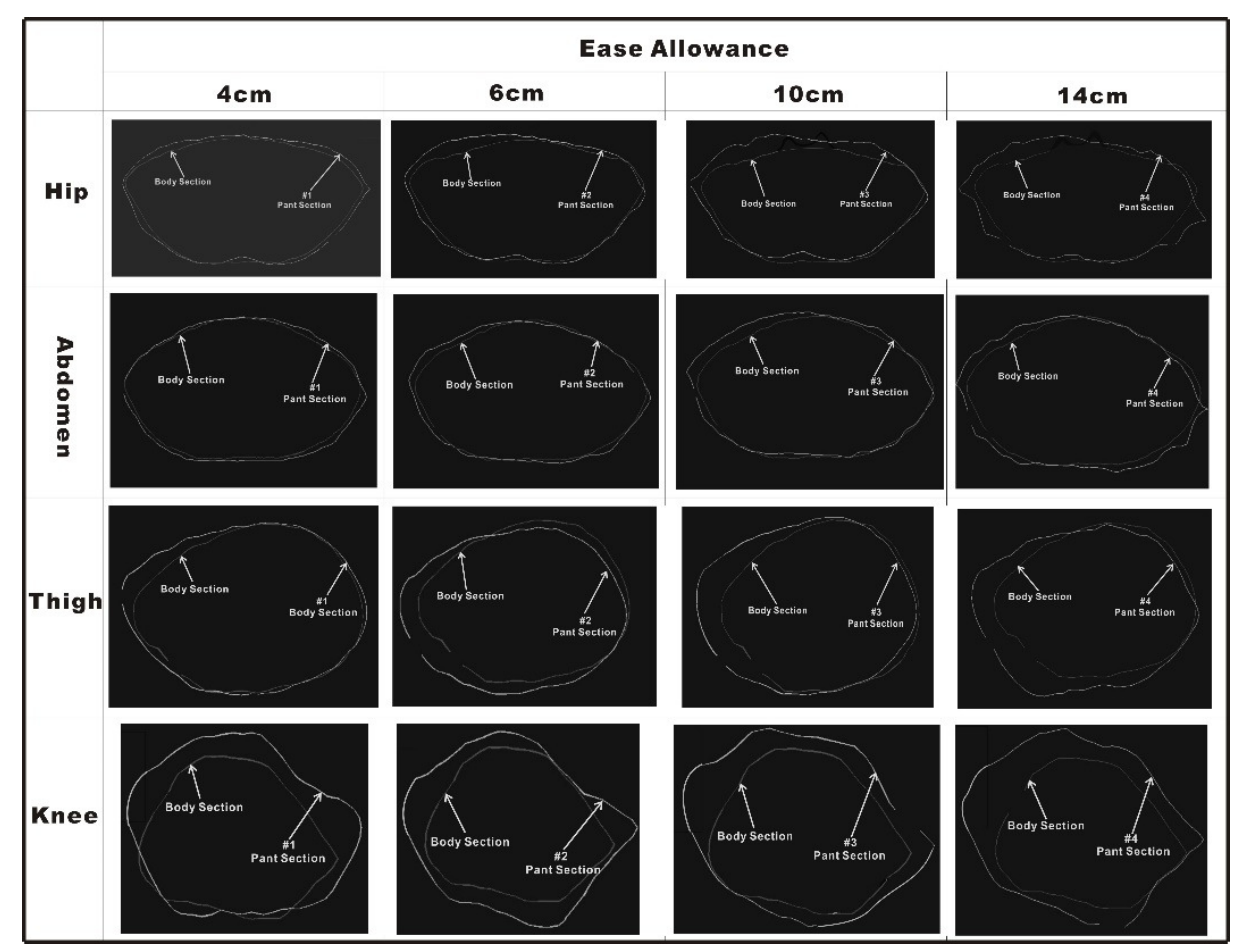

Fig. 5. Pant-body circumferences at different features.

\subsubsection{Measuring of distance ease}

We defined the distance ease as the distance between the pant and body circumferences at a certain postion, such as $\left(A_{1} A_{1}^{\prime}\right)$ in Figure 6 . The method to measure distance ease was carried out as following. A frame was firstly settled down with the help of Width and Thickness of a feature, e.g. hip in Fig. 6. Then some sampling points were determined by trisecting $\overline{\mathrm{OL}}_{s p}$ to get $\mathrm{O}_{1}$ and $\mathrm{O}_{2}$, and then bisecting $\overline{\mathrm{O}_{2} \mathrm{~L}_{\mathrm{sp}}}$ to get $\mathrm{O}_{3}$. Subsequently, vertical lines were made through $\mathrm{O}_{1}, \mathrm{O}_{2}, \mathrm{O}_{3}$ separately which intercept with the body cross-section and the pant cross-section at $A_{1} / \mathrm{A}_{1}^{\prime}, A_{2} / \mathrm{A}_{2}^{\prime}, A_{3} / \mathrm{A}_{3}^{\prime}, B_{1} / \mathrm{B}_{1}^{\prime}, B_{2} / \mathrm{B}_{2}^{\prime}, B_{3} / \mathrm{B}_{3}^{\prime}$. Thus distance ease data, e.g., $\overline{A_{0}^{\prime} A_{0}}, \overline{A_{1}^{\prime} A_{1}}, \overline{A_{2}^{\prime} A_{2}}, \overline{A_{3}^{\prime} A_{3}}, \overline{B_{0}^{\prime} B_{0}}, \overline{B_{1}^{\prime} B_{1}}, \overline{B_{2}^{\prime} B_{2}}, \overline{B_{3}^{\prime} B_{3}}, \overline{L^{\prime} L_{s p}}$, can be measured and recorded. The same procedure is applied to the other side of the pant-body circumferences to get the final mean of distance ease.

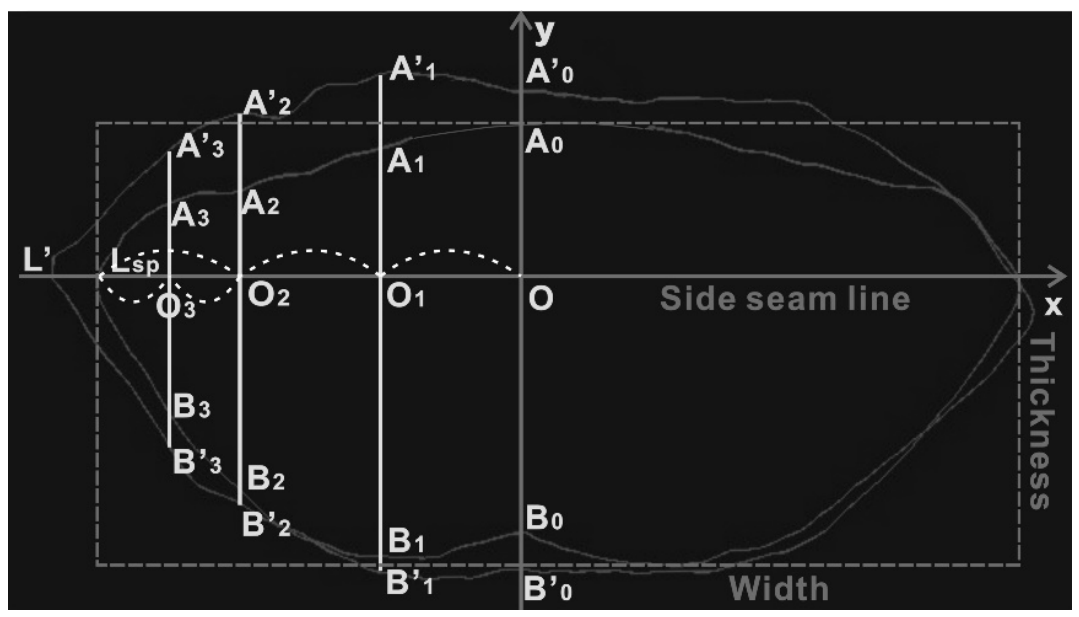

\subsubsection{Regression Analysis}

Based on a basic pant pattern, if the distance ease can be added to exact positions in the pattern structure, then it becomes easier to develop an personalized pattern of pants automatically. Therefore, the correlation analysis was done to explore the relationship between the distance ease and the ease allowance. Let $\varphi$ present the distance ease at one sampling point and $\omega$ the ease allowance of a pant. The regression equations were obtained after scatter diagram test and correlation analysis, the result for the distance ease at selected sampling points and ease allowance given were shown in Table 6 . 
Table 6. Result of regression analysis for distance ease and ease allowance.

\begin{tabular}{|c|c|c|c|c|}
\hline Distance Ease & Regression & $\varphi(\mathrm{cm})$ & $\left.\alpha()^{\circ}\right)$ & $\Delta_{l}(\mathrm{~cm})$ \\
\hline$\overline{A_{0}^{\prime} A_{0}}$ & $\phi=-0.651+0.302 \omega-0.003 \omega^{2}$ & 2.07 & 25 & 0.9 \\
\hline$\overline{A_{1}^{\prime} A_{1}}$ & $\phi=0.349+0.149 \omega+0.002 \omega^{2}$ & 2.04 & 9 & 0.3 \\
\hline$\overline{A_{2}^{\prime} A_{2}}$ & $\phi=0.714+0.038 \omega+0.003 \omega^{2}$ & 1.39 & 15 & \\
\hline$\overline{A_{3}^{\prime} A_{3}}$ & $\phi=4.662-1.115 \omega+0.081 \omega^{2}$ & 1.61 & 26 & 0.7 \\
\hline$\overline{B_{1}^{\prime} B_{1}}$ & $\phi=3.957-0.968 \omega+0.065 \omega^{2}$ & 0.78 & 26 & 0.4 \\
\hline$\overline{B_{2}^{\prime} B_{2}}$ & $\phi=0.859-0.12+0.004 \omega^{2}$ & 0.26 & 14 & 0.1 \\
\hline$\overline{B_{3}^{\prime} B_{3}}$ & $\phi=-1.035+0.46 \omega-0.021 \omega^{2}$ & 0.87 & 24 & 0.6 \\
\hline$\overline{L^{\prime} L s p}$ & $\phi=-0.251+0.077 \omega+0.01 \omega^{2}$ & 1.52 & 41 & 1.2 \\
\hline
\end{tabular}

2.2.3 Model of Distance Ease-Increment

It is further necessary to make clear that the relationship between the increment of a pattern and the distance ease. So we proposed a model called DE-Increment (Distance Ease-Increment) to reflect this relationship.

The pant-body circumferences were abstracted as two circles, as shown in Fig. 7. The increments for the pattern can be described as: $\Delta \mathrm{L}=\mathrm{L}_{\mathrm{c}}-\mathrm{L}_{\mathrm{b}}=\mathrm{DE} * \alpha$. In this model, $\Delta \mathrm{L}$ means the increment of the pant pattern in girth, e.g., hip, caused by distance ease, and $\alpha$ is the angel between two sampling points. The method to measure angle $\alpha$ is shown in Fig. 8. In the narrow space, the distance ease and the radial between the pants and body can be considered approximately equal.

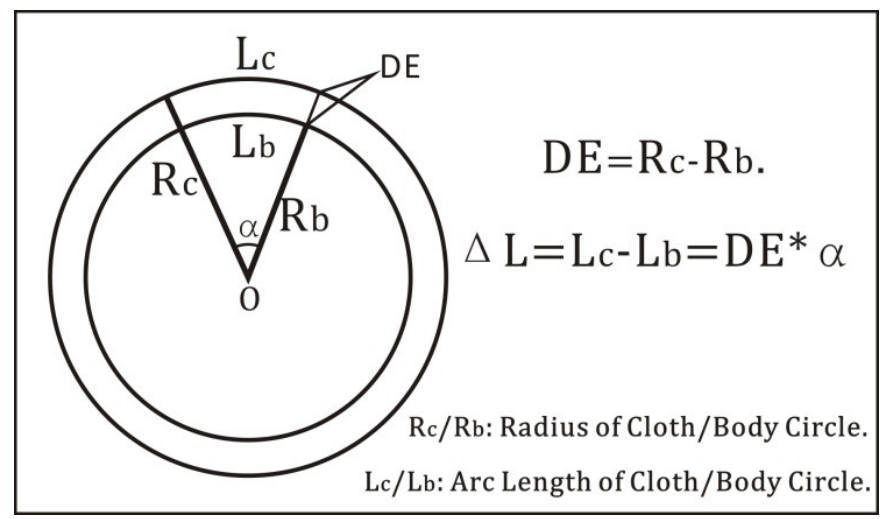

Fig. 7. DE-Increment model.

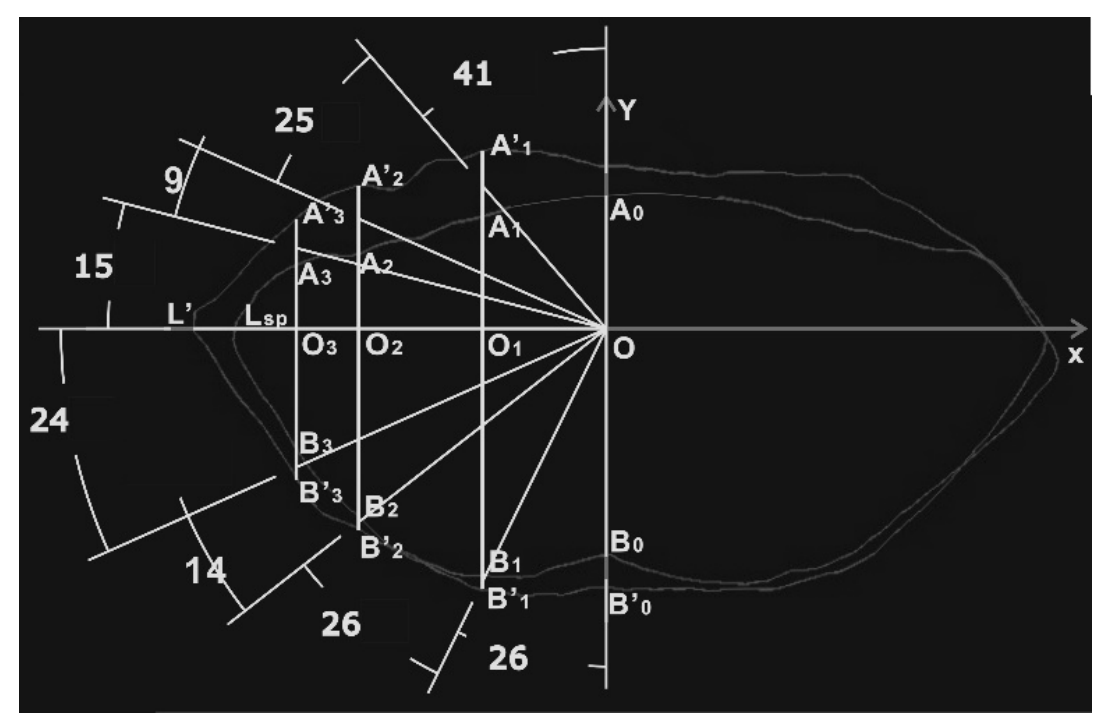

Fig. 8. Method to measure angle $\alpha$. 
Once the ease allowance $\omega$ were given, the distance ease $\varphi$ could be got by regression equations. By applying the distance ease $\varphi$ and the angel $\alpha$ into the $D E$-Increment model, the increment of the pattern $\Delta \mathrm{L}$ at different sampling positions can be calculated. Table 6 shows the increments $\Delta \mathrm{L}$ of the pattern of pant \#3 at different sampling points on the hip girth.

\subsection{Development of a personalized pant pattern by altering}

Once the increments of a pattern obtained, it can be used to alter the patter for purpose of personal fit. First, a basic pant pattern can be generated according to the relationship of body and apparel pattern illustrated in Fig. 1. The length of Front/Back Waist can be calculated using regression equation in Table 3 in an individual way, and other elements can also be calculated out by similiar ways. The basic pattern in Fig. 9 includs an ease allowance of $1 \mathrm{~cm}$ at waist and have no allowance at hip. This pattern fits tightest and reflects the body shape well. Secondly, the increments $\Delta_{l}$ in Table 6 were added to their locations in the front and back patterns respectively. This process was shown in Fig. 9 (b). The directions of the increments are determined to ensure a balanced pattern. There were totally $9.2 \mathrm{~cm}$ increments in the hip line. Another $8.8 \mathrm{~cm}$ increment to the thigh line was added with the same method. The smooth lines were made to pass through all of the adjusted points, and the final patterns were shown in Fig. 9 (c).

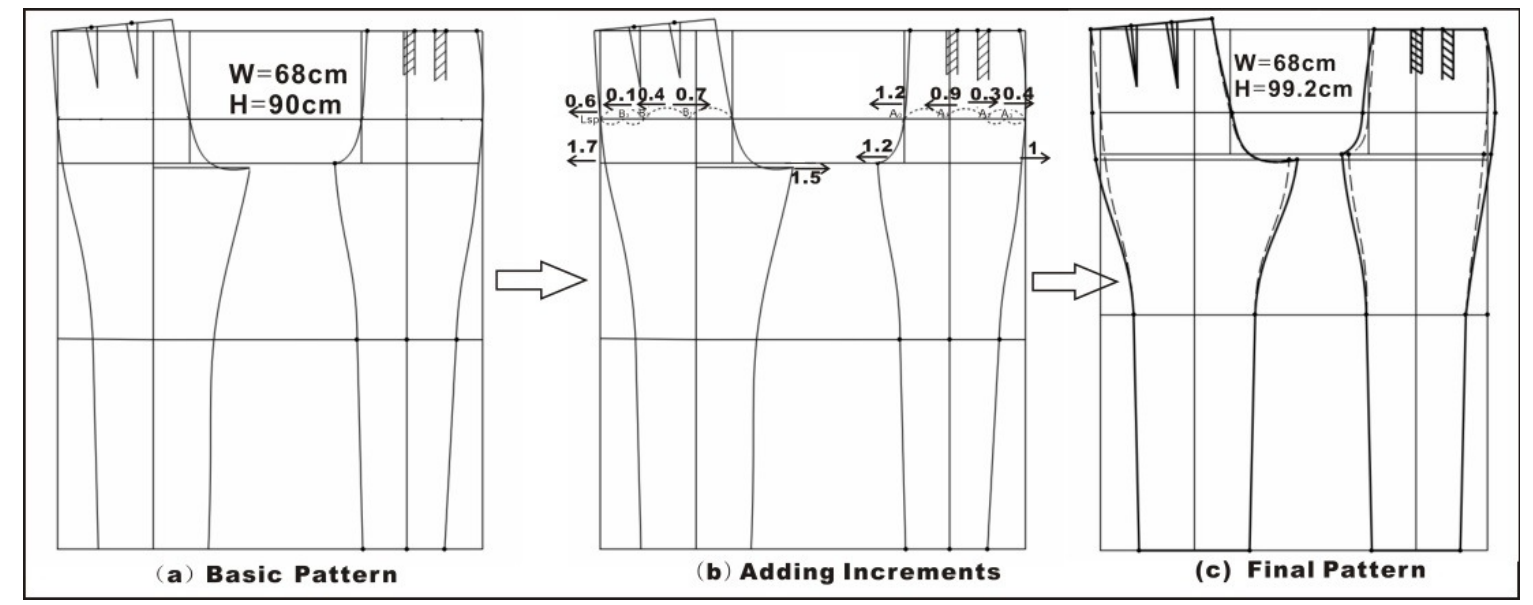

Fig. 9 Alter a basic pattern to develop a personalized pant pattern.

\section{Conclusions}

A method to develop a personalized pattern of pant was studied in this paper. There were three main parts to complete the purpose: 1) realization of individuality of a body. In this part, a method to classify the parameter which reflects the relationship between a feature and an element of pattern plays the most important role. 2) description of distribution of ease allowance at characteristic features. This part deals with the issue that how an ease allowance distribute at different characteristic features, and the relationship among ease allowance, distance ease and increment of a pattern were discussed especially. And 3) a method to develop a personalized pant pattern by altering a basic pattern using the results from the above two parts. The main innovation of this paper are the method to classifying a feature to express individuality of body in the structure of pattern and increment at a certain feature based on a basic pattern caused by an ease allowance given by a designer. It showed a complete way to develop a personalized pant pattern from 3D body scanning data.

\section{Acknowledgments}

The authors would like to thank the financial support of the Natural Science Foundation of Jiangsu Province ( Grants No. BK20151191) and the Fundamental Research Funds for the Central Universities

(Grants No. JUSRP51735B). This study was also supported by Jiangsu Intangible Cultural Heritage Research Base.

\section{References}

[1] C. L. Istook, "Enabling mass customization :Computer-driven alteration methods," Int. J. Cloth. Sci. Technol., vol. 14, no. 1, pp. 61-76, 2002, https://doi.org/10.1108/09556220210420345. 
[2] B. Xu, W. Yu, T. Chen, and Y. Huang, "Three-dimensional Technology for Apparel Mass Customization: Part II: Human Body Modeling from Unorganized Range Data," J. Text. Inst., vol. 94, no. 1-2, pp. 81-91, Jan. 2003, http://dx.doi.org/10.1080/00405000308630596.

[3] B. G. Xu, Y. Huang, W. Yu, and T. Chen, "Three-dimensional body scanning system for apparel mass customization," Opt. Eng., vol. 41, no. 7, pp. 1475-1479, 2002, doi:10.1117/1.1478700.

[4] S. Kwon, S. Ha, and C. Kowal, "How online self-customization creates identification: Antecedents and consequences of consumer-customized product identification and the role of product involvement," Comput. Human Behav., vol. 75, pp. 1-13, 2017, https://doi.org/10.1016/j.chb.2017.04.051.

[5] J. Su, B. Gu, G. Liu, and B. Xu, "Determination of distance ease of pants using 3D scanning data," Int. J. Cloth. Sci. Technol., vol. 27, no. 1, pp. 47-59, 2015, https://doi.org/10.1108/IJCST-08-2013-0096. [6] J. Su, G. Liu, and B. Xu, "Development of individualized pattern prototype based on classification of body features," Int. J. Cloth. Sci. Technol., vol. 27, no. 6, pp. 895-907, 2015, https://doi.org/10.1108/IJCST-11-2014-0136.

[7] Y. Yang, W. Zhang, and C. Shan, "Investigating the development of digital patterns for customized apparel," Int. J. Cloth. Sci. Technol., vol. 19, no. 3/4, pp. 167-177, 2007, https://doi.org/10.1108/09556220710741632

[8] H. Q. Huang, P. Y. Mok, Y. L. Kwok, and J. S. Au, "Block pattern generation: From parameterizing human bodies to fit feature-aligned and flattenable 3D garments," Comput. Ind., vol. 63, no. 7, pp. 680691, Sep. 2012, https://doi.org/10.1016/i.compind.2012.04.001.

[9] Y. Zhong and B. Xu, "A physically based method for triangulated surface flattening," CAD Comput. Aided Des., vol. 38, no. 10, pp. 1062-1073, 2006, https://doi.org/10.1016/i.cad.2006.05.002.

[10] J. Li and G. Lu, "Modeling 3D garments by examples," CAD Comput. Aided Des., vol. 49, pp. 2841, 2014, https://doi.org/10.1016/i.cad.2013.12.005.

[11] C. Wang, Y. Wang, and M. Yuen, "Feature based 3D garment design through 2D sketches," Comput. Des., vol. 35, pp. 659-672, 2003, https://doi.org/10.1016/S0010-4485(02)00091-X.

[12] Y. Meng, P. Y. Mok, and X. Jin, "Computer aided clothing pattern design with 3D editing and pattern alteration," Comput. Des., vol. 44, no. 8, pp. 721-734, Aug. 2012, https://doi.org/10.1016/i.cad.2012.03.006.

[13] S. Petrak and D. Rogale, "Systematic representation and application of a 3D computer-aided garment construction method Part I: 3D garment basic cut construction on a virtual body model," Int. J. Cloth. Sci. Technol., vol. 18, no. 3, pp. 179-187, 2006, https://doi.org/10.1108/09556220610657943.

[14] N. Masaru and G. Yuan, Human Body and Garment. Beijing: China Textile \& Apparel Press, 2003. [15] Zhang, Aiping, Wang, Yunyi, and YAO, Yi, "Study on relationships between garment's distance ease distributions at bust section," J. Text. Res., vol. 33, no. 6, pp. 76-80, 2012.

[16] J. Su et al. "Distribution of ease allowance of pants and it's application in individualized pattern making, "J. Cloth. Res., vol.1, no. 5, pp.487-491, 2016. 